tion hat, sondern vielmehr einem feuchten Teige gleicht, der sich in Gührung befindet. (Aus d. Compt. rend., durch chem. Centralbl. 1873. S. 769. Polyt. Notizbl. v. Böttger. 1874. p. 58.).

C. Sch.

\title{
Vertheilung des Kali's und Natron's in den Gewächsen.
}

E ug. Peligat liefert die Fortsetzung seiner Arbeiten*) über die Betheiligung der Alkalien, des Kali's und Natron's an der Production der Gewächse, in denen er gezeigt hat, dass die Asche einer grossen Anzahl Culturpflanzen reich an Kali und frei von Natron ist. Die Abwesenheit dieses Alkali's beruhe jedenfalls nicht auf dem Fehlen der Natronsalze, besonders des Chlornatriums im Boden und den Düngemitteln, da sich neben diesen natronfreien Pflanzen andere entwickelten, welche einen bedeutenden Natrongehalt aufzuweisen batten.

Diese Thatsachen wurden anfangs bestritten. Man habe eingewendet, dass, wenn die Culturgewächse auf salzhaltigem Boden, z. B. in der Nähe des Meeres wüchsen, sich das Verhältniss ganz anders gestalten würde. Verf. glaubt jedoch bewiesen zu haben, dass das in der Asche dieser Pflanzen gefundene Natron nicht aus dem Boden stammt, sondern wegen des Natrongehalts der Luft auf der Oberfläche der Pflanzen befindlich gewesen ist.

Man habe ferner geltend gemacht, das Chlornatrium sei vielleicht wegen der zu grossen Hitze beim Veraschen verflogen. Auf diesen Einwurf glaubt Verf. schon geantwortet zu haben, indem er oft darauf hingewiesen, dass die Veraschung bei möglichst niedriger Temperatur vorzunehmen sei, selbst auf die Gefahr hin, nicht ganz weisse Aschen zu erhalten, und theilt zugleich das Resultat einiger zur Feststellung etwaiger Versuchsfehler angestellten Controlversuche mit.

Dieselben wurden in der Weise ansgeführt, dass man $10 \mathrm{~g}$. lufttrockne Maulbeerblätter mit einer Lösung von $0,1 \mathrm{~g}$. Chlornatrium anfeuchtete und nach dem Trocknen, Veraschen und Auslangen der Asche den Chlorgehalt bestimmte.

Andererseits wurde in der Asche einer gleichen Menge lufttrockner Maulbeerblätter die Menge des an Kalium

*) Annal. de Chimie et de Phys, 4e série tXII. p. 431. tXVIII. p. 431 , t XIII. p. 406. 
gebundenen Chlor's bestimmt und gefunden, dass der, nach Abzug dieser von der oben genannten Menge, bleibende Rest an Chlor entsprach $0,1 \mathrm{~g}$. Chlornatrium.

Viele zu gleichem Zweck angestellte Versuche ergaben dieselben Resultate.

Verf. theilt dann seine nene Arbeit mit, welche die Frage beantworten soll:

"Nimmt eine während ihrer ganzen Entwicklungszeit mit einer Lösung von Chlornatrium oder salpetersaurem Natron begossene Pflanze eine gewisse Menge Natron auf, und entzieht sie dem Boden andere Elemente als unter gleichen Bedingungen cultivirte Pflanzen derselben Art, welche entweder mit gewöhnlichem Wasser, mit Kalisalz-oder MagnesiasalzLösung begossen werden?"

Zur Lösung dieser Frage wurde im Juli 1873 eine gleiche Anzahl Bohnen in 12 grosse Blumentöpfe gepflanzt, welche 13 bis 15 Liter fassten und $20-23 \mathrm{Ko}$ durch Umschaufeln möglichst gut gemischte, poröse Erde enthielten. Eine summarische Analyse dieser getrockneten Erde ergab folgende Zusammensetzung:

Organische Substanz

Carbonate der Kalkerde und der Magnesia 11,6

Thonerde

Sand

$$
\begin{array}{r}
20,0 \\
60,2 \\
\hline 100,0
\end{array}
$$

Dieselbe enthielt übrigens die in gewöhnlicher Gartenerde durchschnittlich enthaltenen Mengen Kali, Phosphorsäure, Eisenoxyd etc.

Jeder Versuch wurde doppelt angestellt; und erhielten die Töpfe Nr. 1 und 2 jeder 10 Liter Seinewasser; Nr. 2 und 3 je 5 Liter desselben Wassers, welche 1 g. Chlornatrium enthielten, und später noch 5 Liter Wasser, in welchen $2 \mathrm{~g}$. Chlornatrium gelöst waren; Nr. 5 und 6, 15 g. Chlorkalium, $\mathrm{Nr} .7$ und 8, 15 g. salpetersaures Natron; Nr. 9 und 10, $15 \mathrm{~g}$. salpetersaures Kali und Nr. 11 und 12, $15 \mathrm{~g}$. schwefelsaure Ammon - Magnesia. Diese verschiedenen Salze waren in den oben genannten Mengen Wassers gelöst.

Von Anfang an zeigte sich der schädliche Einfluss des Chlornatriums. Die damit begossenen Pflanzen hatten gelbe Blätter und contrastirten mit den dunkelgrïnen, auderen Pflanzen; die Blüthenentwicklung ging langsam vor sich, und starb eine Pfanze ganz ab. Nach dem Keimen der Bohnen waren in jedem Topfe 4 Pflanzen gelassen, und erntete man 
von jedem Topfe 75-100 g. Trockensubstanz (Stengel Blatter und Samen), während das Gewicht der trocknen Chlornatrium-Pflanzen nur $55 \mathrm{~g}$. betrug. ${ }^{*}$ )

Die Töpfe standen im Freien ohne Bedachung. Die Ernte wurde am 14. September vorgenommen.

Das Gewicht der bei sehr niedriger Temperatur hergestellten Asche betrug $10-14 \%$ der Trockensubstanz und trennte man die löslichon Substanzen derselben (Kali zuweilen Natronsalzc) von den unlöslichen (Kieselsäure, Carbonate und Phosphate des Calciums und Magnesiums, Eisenoxyd, Manganoxyd und Kohle) durch Auslaugen.

der Aschen:

Folgende Tabelle zeigt die procentische Zusammensetzung

Nr. 1 u. $2 . \quad 3$ u. 4.5 und 6.7 und 8.9 und 10.11 und 12

Seinewas- Chlorna- Chlor- Salpeters. Salpeters. Schwefels.

ser ohne trium. kalium. Natron. Kali. Anmon-

Kieselsäure

Zusatz.

Kalk

7,2

$$
15,2
$$

13,6

10,4

Magnesia

Phosphorsaure 11,2

$29,6 \quad 26,5$

22,7

12,1

13,0

Magnesia.

Magnesia

9,5

8,5

21,8

18,5

24,9

$9,2 \quad 8,2$

11,2

Kohlensäure

Unlösliche Bestand theile.

Kohlensaures

Kali

Chlorkalium

Schwefelsau-

\begin{tabular}{|c|c|c|c|c|c|}
\hline 2,0 & 2,4 & 1,6 & 1,5 & 0,9 & 2,0 \\
\hline 9,0 & 6,1 & 11,8 & 17,1 & 17,8 & 6,9 \\
\hline 59,0 & 59,7 & 58,2 & 60,0 & $\begin{array}{l}57,5 \\
\vdots\end{array}$ & 58,0 \\
\hline 33,0 & 6,6 & 4,5 & 28,3 & 28,2 & 23,8 \\
\hline 0,6 & 26,5 & 27,2 & 3,4 & 4,7 & 4,7 \\
\hline
\end{tabular}

res Kali.

Lösliche Bestand theile.

\begin{tabular}{lll}
7,4 & 7,2 & 10,1 \\
\hline 41,0 & 40,3 & 41,8
\end{tabular}

$\begin{array}{ll}8,3 & 9,6 \\ 40,0 & 42,5\end{array}$

Verf. sagt nun: ..Vergleicht man die Zahlen dieser Analysen, so sicht man, dass das Verhältniss, welches zwischen löslichen und unlöslichen Bestandtheilen bestcht, wenig variirt; die löslichen schwankon zwischen 40 und 42,5\% der Asche. Dieselbe Bemerkung kann man hinsichtlich der unlöslichen Bestandtheile machen; die Unterschicde sind nicht scharf

*) In einer anderen Versuchs - Reihe keimten Bohnen, welche mit Wasser begossen waren, welches $1 \mathrm{p}$. m. Chlornatrium enthielt, selbst dann nicht, als das salzhaltige durch gewöhnliches Wasser ersetzt wurde. 
genug, um daraus irgend welche Schlüsse über die Wirkung der verschiedenen Salze zu ziehen.

Nichts desto weniger ist darauf aufmerksam zu machen, dass die mit schwefelsaurer Ammon-Magnesia begossenen Pflanzen nicht mehr Magnesia enthalten, als die mit reinem Wasser begossenen.

Das Studium der löslichen Bestandtheile bietet dagegen grosses Interesse. Nach Verhältniss der Oberfläche der Töpfe beträgt die Menge der zugesetzten Salze, auf eine Hectare berechnet, $3000 \mathrm{Ko}$. . Indessen sieht man aus der Tabelle, dass Chlornatrium und salpetersaures Natron von den Pflanzen völlig vernachlässigt sind; keine der Aschen enthält Natron.

Dagegen haben die mit den Natronsalzen begossenen Pflanzen das im Boden enthaltene Kali aufgenommen, und glaubt Verf. jenen beiden Salzen nicht alle Wirkung absprechen zu dürfen, weil sich dicselben wahrscheinlich mit anderen Basen (Kali oder Kalk) umsetzten und auf diese Weise sowohl Chlor wie Salpetersäure den Pflanzen zu Gnte kämen, wie solches aus dem grossen Chlorkaliumgehalte der mit Chlornatrium begossenen Pflanzen hervorgehe.

Uebrigens sei bemerkenswerth, dass die den Töpfen 5 u. 6 , sowie 9 u. 10 zugesetzten Kalisalze den Gehalt der Aschen an diesem Alkali nicht merklich vergrössert haben. Für Pflanzen und Thiere finde sich die Assimilations-Fähigkeit wahrscheinlich auf sehr enge Grenzen beschränkt. Beide könne man nicht mehr absorbiren lassen, als sie zu ihrer Entwicklung nöthig haben. So wäre in diesen Versuchen der Boden mit der zur Vegetation nöthigen Menge Kali, Kalk, Magnesia, Phosphorsäure, Schwefelsäure, Eisen, Mangan und Kieselsäure versehen gewcsen, die Pflanzen hätten daher aus den Lösungen nichts aufgenommen.

„Wenn das Chlor, welches sich in beträchtlicher Menge in den mit Chloralkalien begossenen Pflanzen findet, eine Ausnahme macht, so kann das daher kommen," sagt Verf., dass der Boden in Antange des Ver'suchs nur eine der absorbirenden Kraft der Ptanze nicht entsprechende Menge Chlor enthielt. Auch bin ich geneigt, entgegen den Forderungen, welche man aus einer sehr grossen Anzahl mehr oder weniger gut ausgeführter Analysen ziehen kann, anzunehmen, dass wenn die Düngemittel die Fähigkeit haben, hinsichtlich des Gewichts der Ernten, die landwirthschaftlıche Production zu steigern, sie sehr wonig die Natur und Menge der Mineral- 
bestandtheile, welche sich in der als Individuum betrachteten Pflanze anhäufen, modificiren.

Die Ansichten Che vreuls über die ergänzenden Düngemittel sind den eben ausgesprochenen gleich, mit der Ausnahme jedoch, dass für die meisten Culturgewächse das Natrium küzftig in der Liste dieser Düngemittel zu streichen ist." "

Nach einer ausfuihrlichen Beschreibung der angewendeten analytischen Methoden wirft Verf. die Frage auf, wie es komme, dass eine mit Chlornatrium begossene Pflanze das Chlor zurïckhalte und das Natrium nicht absorbire? Hierüber könne man nur Hypothesen aufstellen, weil die Frage zu verwickelt, und das Material zur Lösung derselben zu ungenügend sei. Nichts desto weniger dürfe man nach dem mit Chlornatrium angestellten Versuche annehmen, dass sich dieses mit im Boden vorhandenen schwefelsauren Kalk umsetze in schwefelsaures Natron, welches die Pflanze nicht aufnimmt und in Chlorcalcium, welches aufgenommen wird. Nichts beweise bis jetzt, dass das Chlorcalcium nicht eine in der Pflanzenproduction nützliche Rolle spiele, wenigstens in den kochsalzreichen und trotzdem gute Ernten liefernden Böden.

Schliesslich weist Verf. noch darauf hin, dass neuerlich durch Schlösing*) das Vorkommen von Chlorcalcium in salz und kalkhaltigen Böden in sehr klarer Weise dargethan ist. (Annal. de chim. et de physique, October 1873.). Dr. F. B.

\section{Eine analoge Berechnungs - und Bezeichnungsart der tesseralen und rhomboëdrischen Krystallgestalten.}

Die Analogie der Flächenlagen beiderlei ist schon längst von Mohs, Naumann u. A. erklärt worden. Für die Berechnung der rhomboëdrischen Gestalten erweist sich nach Krejci vortheilhaft die Betrachtung derselben analog den tesseralen Formen mit Beziehung auf ein dreiachsiges System. Als eine übersichtliche, allgemein anwendbare Bezeichnungsart der Flächenlage könnten die hexö̈dischen Flächen mit h, die octoïdischen mit $o$ und die dodecoïdischen mit $d$ bezeichnet werden. Die Parameterverhältnisse jeder Fläche lassen sich dann als diesen Buchstaben angehängte Indices bezeichnen.

*) Compt. rend. t. LXXIII. p. 1326. 